Fourth International Symposium on Physics of Fluids (ISPF4)

International Journal of Modern Physics: Conference Series

Vol. 19 (2012) 424-434

(C) World Scientific Publishing Company

DOI: $10.1142 / \mathrm{S} 2010194512009026$

\title{
THERMAL PERFORMANCE OF WIND TURBINE POWER SYSTEM'S ENGINE ROOM
}

\author{
ZHILI LIU, YANLONG JIANG, NIANYONG ZHOU, HONG SHI, NA KANG AND YU WANG \\ Man-Machine-Environment Engineering, College of Aerospace Engineering, \\ Nanjing University of Aeronautics and Astronautics, Nanjing 210016, China, \\ liuzhilichery@hotmail.com
}

\begin{abstract}
Greatly expanded use of wind energy has been proposed to reduce dependence on fossil and nuclear fuels for electricity generation. For wind turbine power generation, as a mature technology in the field of wind power utilization, its large-scale deployment is limited by the cooling technology. Therefore, the temperature distribution of the wind turbine power generation is a key issue for the design of the cooling system. It is because the characteristics of cooling system have a great effect on the performance of the wind turbine power generation. Based on some assumptions and simplifications, a thermal model is developed to describe the heat transfer behavior of wind turbine power system. The numerical calculation method is adopted to solve the governing equation. The heat generation and heat flux are investigated with a given operating boundary. The achieved results can be used to verify whether the cooling system meets the design requirements. Meanwhile, they also can reveal that among the influencing factors, the meteorological conditions, generated output and operation state as well seriously influence its thermal performance. Numerical calculation of the cooling system enables better understanding and results in performance improvement of the system.
\end{abstract}

Keywords: Wind turbine; heat transfer; numerical calculation.

\section{Introduction}

Energy saving and $\mathrm{CO}_{2}$ emission reduction are among the most important issues attracting international attention. An increasing awareness of these issues has prompted the application of the renewable energy (RE) sources especially wind energy for electricity generation. The cooling system of wind turbine power generation plays an important role in ensuring the wind turbine power system operating reliability and stablility ${ }^{1-3}$.

The cooling system supplies cold energy for the whole wind turbine power system, responsible for carrying away the heat produced by the wind turbine power system ${ }^{4}$. Meanwhile, different components with the different boundary condition have heat transfer with the air of cabin, which results in the high temperature of cabin air. Inhomogeneous temperature and unreasonable design of the cooling system would make the air of cabin exceed the designed value. More serious, some unpredictable 
consequences may occur since the some components in cabin are temperature-sensitive assembly such as electrical equipment ${ }^{5-7}$. In view of these facts, the thermal performances inside the cabin should be measured at the initial stages of the design. Hence, this work would have significant impacts on the comprehensive performance of wind turbine power generation besides proper system designs.

The investigation in this paper focuses on the thermal performance of a liquid cooling system of wind turbine power generation by connected simulation of convection, conduction and radiation. Over the past years, many researchers and experts in the wind turbine field have devoted considerable efforts to study the operation of liquid cooling system. Yuan et $a l^{8}{ }^{8}$ studied different cooling systems of the wind turbine wind generation. They found that the high-power wind turbine power generation has a preference for liquid cooling system. Similar work was done by $\mathrm{Ni}^{9}$, who studied the liquid cooling system of the generator cooling and gave some introduction of the basic control strategies. In these studies, the heat transfer between the key components and the air inside cabin and solar radiation on heat transfer was not considered. But for the realistic performance estimation, the thermal contact between the surface of the key components and the air of the cabin, the temperature distribution caused by solar radiation is of paramount importance to conduct the cooling system and optimize the thermal performance.

The objective of present work is to numerically analyze the thermal performances of the wind turbine power generation. In this paper the numerical calculation method is used to investigate the characteristics of heat load of the cabin. The factors such as geometric configuration, physical parameters, the meteorological conditions and operation state that impact thermal performance of the wind turbine power generation would be considered.

\section{Mathematical Model}

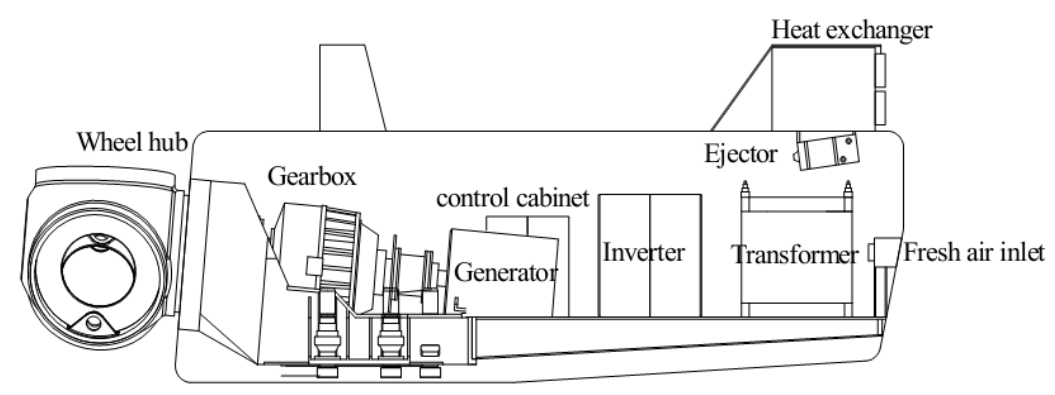

Fig. 1. Schematic diagram of the wind turbine cabin.

The core components of the wind turbine power generation are concentrated layout. The sketch of the wind turbine cabin is shown in Fig. $1^{10}$. The wind turbine carbine consists of gearbox, generator, inverter, transformer, control cabinet, and ejector mainly can be seen in Fig. 1. They are very important heat source in the inner cabin, while the solar radiation, the heat load of the fresh air used to maintain the positive pressure of cabin and the 
convective heat transfer between the cabin and external environment contributes to the external heat source of the cabin.

There are several typical heat transfer behaviors proceeding in the wind turbine cabin, such as solid conduction, gas conduction, and gas natural convection in the inner cabin and radiation heat transfer between the surface of the core components and internal surface of the cabin. The heat transfer mechanism of the wind turbine cabin is shown in Fig. 2. In order to simplify the mathematical model, the following assumptions are considered ${ }^{11-14}$ :

(1) there are many components in the cabin of wind turbine and each component's surface is isothermal; (2) the heat transfer process between the outer wall of the cabin and the external environment can be assumed to be flat flow; (3) the specific heat and thermal conductivity of air and different components are constant.

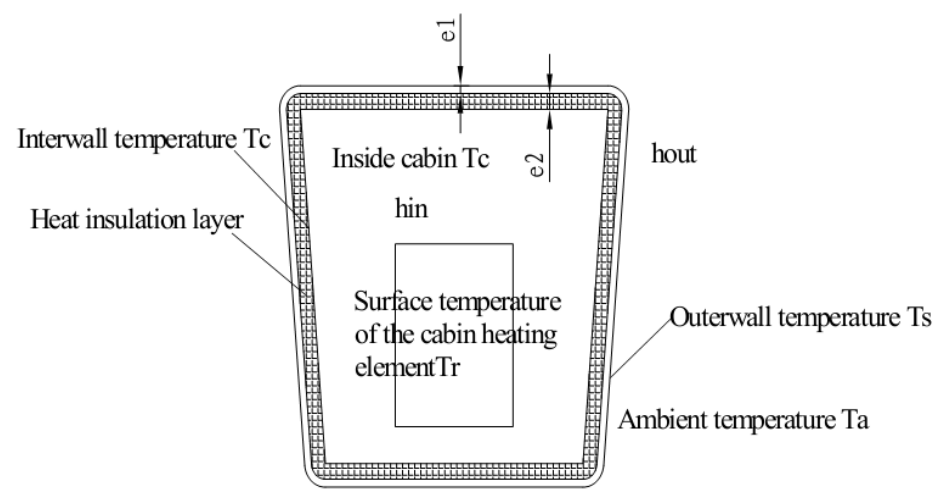

Fig. 2. The heat transfer mechanism of the wind turbine.

\subsection{Governing equations}

Based on the above assumptions, a model of thermal analysis is developed to describe the physical phenomena. Fig. 3 gives the physical model involved in the operation of the solar radiation, the inner heat source, the fresh air load and the cooling coils load. Taking the cabin air temperature as variable, the thermal equation of the system shown in Fig. 3 is given by

$$
m c_{p} \frac{d T}{d t}=Q_{r}+Q_{j}+Q_{e}+Q_{x},
$$

where $Q_{r}$ is the inner heat source load of cabin, it consists of the gearbox heat dissipating $Q_{r 1}$, the generator heat dissipating $Q_{r 2}$, the inverter heat dissipating $Q_{r 3}$, the transformer heat dissipating $Q_{r 4}$, the pump of the cooling system heat dissipating $Q_{r 5}$, the fan of the ejector heat dissipating $Q_{r 6}$, the fan of the positive pressure system heat dissipating $Q_{r 7}$, the control cabinet heat dissipating $Q_{r 8}$ and cooling cabinet heat dissipating $Q_{r 9}$ (see Fig. 3); $Q_{j}$ is the thermal load emitted from the cooling coils; $Q_{e}$ is the heat load absorbed from the external environment; $Q_{x}$ is the thermal load from ventilation, respectively. 


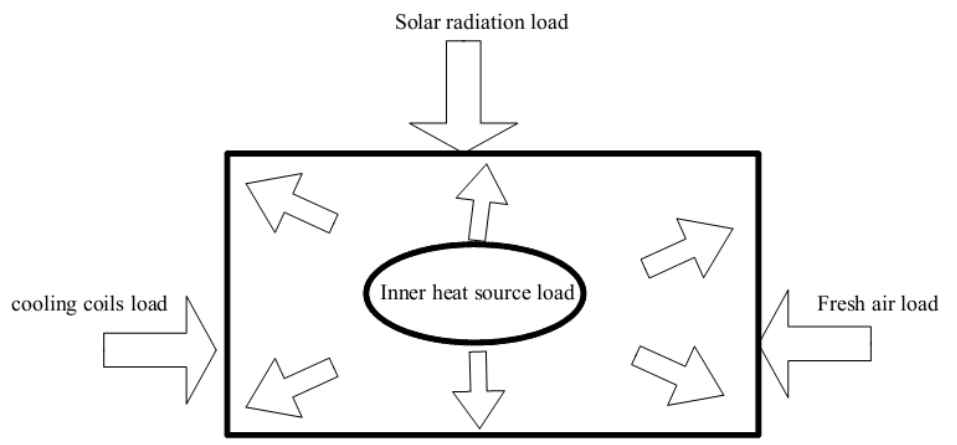

Fig. 3. Schematic diagram of the physical model.

The above loads can be given depending on Fourier's law and thermal radiation laws:

$$
\begin{array}{lll}
Q_{r}=Q_{r 1}+Q_{r 2}+Q_{r 3}+Q_{r 4}+Q_{r 5}+Q_{r 6}+Q_{r 7}+Q_{r 8}+Q_{r 9} \\
Q_{r 1}=k_{i n} A_{1}\left(t_{1}-t_{c}\right) & Q_{r 2}=k_{i n} A_{2}\left(t_{2}-t_{c}\right) & Q_{r 3}=k_{i n} A_{3}\left(t_{3}-t_{c}\right) \\
Q_{r 4}=k_{i n} A_{4}\left(t_{4}-t_{c}\right) & Q_{r 5}=P_{5}\left(1-\eta_{5}\right) & Q_{r 6}=P_{6}\left(1-\eta_{6}\right) \\
Q_{r 7}=P_{7}\left(1-\eta_{7}\right) & Q_{r 8}=Q_{c} & Q_{r 9}=P_{b 9} l_{19}+P_{b 9} l_{29} c+P_{s 9} \\
Q_{e}=K A_{e}\left(t_{s}-t_{c}\right) & Q_{s}=c_{p} G \rho_{a}\left(t_{c}-t_{a}\right) & Q_{j}=\dot{m} c_{p}\left(t_{w}-t_{c}\right)
\end{array} .
$$

Substitute Eq. (2) into Eq. (1), then Eq. (3) can be acquired:

$$
\begin{aligned}
m c_{p} \frac{d T}{d t} & =Q_{r}+Q_{j}+Q_{e}+Q_{x} \\
& =\sum_{i=1}^{4} k_{i n} A_{i}\left(t_{i}-t_{c}\right)+\sum_{i=5}^{7} P_{i}\left(1-\eta_{i}\right)+Q_{c} \\
& +P_{b 9} l_{19}+P_{b 9} l_{29} c+P_{s 9}+\dot{m} c_{p}\left(t_{w}-t_{c}\right)+K A_{e}\left(t_{s}-t_{c}\right)+c_{p} G \rho_{a}\left(t_{c}-t_{a}\right)
\end{aligned},
$$

where $t_{1}, t_{2}, t_{3}$, and $t_{4}$ are the surface temperatures of gearbox, generator, inverter, transformer, respectively; $A_{1}, A_{2}, A_{3}$, and $A_{4}$ are the equivalent mean heat transfer coefficients inside cabin; $t_{c}$ is the ambient temperature inside cabin; $P_{i}$ is the power of pump and fan; $\eta_{i}$ is the efficiency of pump and fan; $Q_{c}$ is the thermal load of control cabinet; $\dot{m}$ is the air quantity used to cool the cabin; $c_{p}$ is the specific heat capacity of air; $K$ is the equivalent mean heat transfer coefficient between the cabin and outside environment; $t_{s}$ is outside surface temperature of cabin; $G$ is the air volume of fresh air; $\rho_{a}$ is the density of air; $t_{a}$ is the outside ambient temperatures.

Considering the heat transfer process reaching stead-state, the steady-state thermal balance equation is established as follows: $m c_{p} d T / d t=0$, namely

$$
Q_{r}+Q_{j}+Q_{e}+Q_{x}=0 .
$$


When Eq. (4) is established, the wind turbine power generation can work stably and efficiently.

In this paper, the details on every item of thermal load stated in Eq. (4) will be discussed in following sections. $Q_{r}, Q_{e}$ and $Q_{x}$ will be analyzed numerically, which means that the total heat load of cabin $Q=Q_{r}+Q_{e}+Q_{x}$ will be discussed firstly. Then based on Eq. (4), $Q_{j}$ can be obtained.

\subsection{Equivalent mean thermal coefficient $k_{i n}$}

Heat transfer process between the core components and the air of cabin mainly includes convection and radiation. The quantity of radiation among the components' surface and the air is very small in the cabin when wind turbine is working. Therefore, the radiation is neglected in the calculation, which implies that the thermal coefficient $k_{\text {in }}$ is the convection coefficient between the key components and air. The solution for convection coefficient inside cabin can be determined by the semi-empirical formula and numerical calculation. For the closed cabin space, the average velocity of air is about $1 \sim 2.5 \mathrm{~m} / \mathrm{s}$, the whole heat transfer process is similar to the process conducted in the aircraft cabin or buildings. The method for solving convection coefficient, taking into account the small volume of the cabin, can be determined with reference to the equation which is used in aircraft cabin. It can be written:

$$
k_{i n}=\left(8.23+4.28 v_{c}\right) \sqrt{\rho_{c} / \rho_{0}},
$$

where $v_{c}$ is the averaged velocity of air inside cabin, $\rho_{c}$ is the density of cabin air, $\rho_{0}$ is the density of air at sea level. For the wind turbine power generation, $\rho_{c} \approx \rho_{0}$. Eq. (5) can be rewritten as:

$$
k_{\text {in }}=8.23+4.28 v_{c} .
$$

\subsection{Equivalent mean heat transfer coefficient $K$}

The heat transmission process between the cabin and the outside environments is a combination of several thermal behaviors, such as solid conduction, gas conduction, gas natural convection among the outside spacer and solar radiation. The quantity of gas natural conduction is very small in wind turbine working process in ambient environment, so that gas natural convection is neglected in the calculation. Therefore,

$$
K=\frac{1}{1 / h_{\text {out }}+e_{1} / \lambda_{1}+e_{2} / \lambda_{2}},
$$

where $h_{\text {out }}$ is the mean coefficient of convection heat transfer between the cabin skin and ambient environment; $e_{1}$ denotes the thickness of cabin skin; $e_{2}$ is the thickness of insulating layer; $\lambda_{1}$ and $\lambda_{2}$ represent the heat conductivity coefficients of cabin skin and insulating layer, respectively. 
In above equation, the convection heat coefficient $h_{\text {out }}$ is an important parameter to estimate the thermal performance of wind turbine cabin. In the present study, since the air velocity around cabin is relatively high, and according to the characteristics of the cabin structure, the form of correlation used for the convection heat transfer coefficient of the cabin skin and ambient environment is developed by Chilton-Colburn analogy:

$$
N u=0.332 \operatorname{Re}^{0.5} \operatorname{Pr}^{0.33},
$$

where $\mathrm{Nu}$, Re, and $\operatorname{Pr}$ are Nusselt number, Reynolds number and Prandtl number of process, respectively. Finally, the heat transfer coefficient is determined from:

$$
h_{\text {out }}=N u \frac{\lambda}{x} \text {, }
$$

where $\lambda$ is the heat conductivity coefficient of air at the design condition, $x$ is the characteristic length of cabin.

\subsection{Skin temperature of cabin $t_{s}$}

According to the heat transfer theory, the skin temperature of cabin $t_{s}$, which is mainly influenced by the solar radiation, is a very important parameter in the heat process conducted from the cabin skin to the inside cabin. The solar radiation contributes to the increase of temperature of cabin skin. The governing equation can be expressed:

$$
\begin{aligned}
& h_{\text {out }}\left(t_{a}-t_{s}\right)+q_{s} A_{p} / A \\
& \quad=K\left(t_{s}-t_{c}\right)+0.5 \varepsilon_{o s} \varphi_{o s} \sigma\left(T_{s}^{4}-T_{e f f}^{4}\right)+0.5 \varepsilon_{o g} \varphi_{o g} \sigma\left(T_{s}^{4}-T_{g}^{4}\right),
\end{aligned}
$$

where $q_{s} A_{p} / A$ is the heat flux obtained from the solar radiation, $A_{p} / A$ is ratio between the projected area and total area of fuselage, $A_{p} / A=1 / \pi$ is used in present study; $K\left(t_{s}-t_{c}\right)$ is the heat flux conducted from the cabin skin to the inner of cabin; $\sigma$ is Stefan-Boltzmann's constant, $\sigma=5.67 \times 10^{-8} \mathrm{~W} \cdot \mathrm{m}^{-2} \cdot K^{-4} ; \varepsilon_{o s}$ and $\varphi_{o s}$ are some key coefficients, $\varepsilon_{o s}=0.3$ and $\varphi_{o s}=1 ; T_{\text {eff }}$ is effective thermodynamic temperature of sky, $T_{\text {eff }}=0 K ; T_{g}$ is the averaged surface thermodynamic temperature of ground, $T_{g}=284 K$. The heat load contributed by solar radiation $q_{s}$ is expressed as:

$$
q_{s}=e_{0} I P^{m}
$$

where $e_{0}$ is the absorption coefficient; $I$ is the solar radiation intensity; $P$ represents the atmospheric transparency, which is given to be $0.75 ; m$ is the atmosphere quality.

In the expressions (10) and (11), the parameters $A_{p} / A, \varepsilon_{o s}, \varphi_{o s}, T_{\text {eff }}$, and $T_{g}$ are known. If $t_{a}$ and $t_{c}$ are given, $\mathrm{K}$ can be obtained from Eq. (7). $t_{s}$ can be calculated by iterative method using these expressions. Then the heat flux conducted from the cabin skin to the inside cabin can also be calculated. 


\section{Results and Discussion}

The thermal performance of wind turbine cabin is very important to the working behavior of wind turbine. The generated output, meteorological conditions, geometric and physical parameters influence the thermal performance. That is a key problem for the designer to be aware of such influencing factors. In this section, the influences of parameters on the thermal performance of wind turbine cabin are investigated.

\subsection{Analysis of thermal coefficient}

The convection heat transfer coefficient between the key components inside cabin and the air of cabin, $k_{i n}$, is given in Fig. $4 . k_{i n}$ is related to the velocity of the inner cabin air. It can be seen that the convection heat transfer coefficient increases with the ambient velocity at the same temperature.

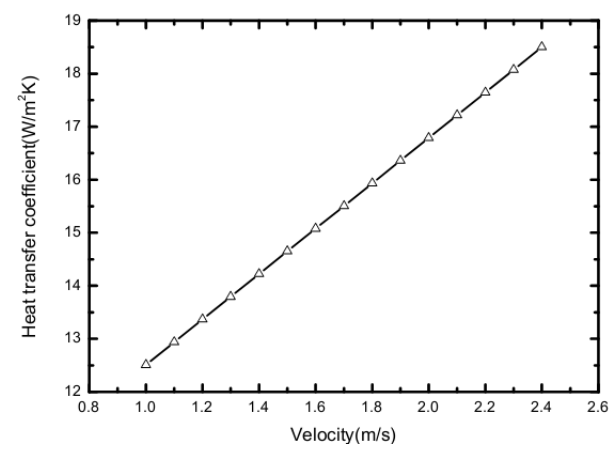

Fig. 4. Variation of inside heat transfer coefficient with the inside air velocity.

Fig. 5 presents the variation of $h_{\text {out }}$ with the ambient velocity. We can obtain that the $k_{\text {in }}$ is related to the velocity of ambient cabin air, and the convection heat transfer coefficient increases with the ambient velocity at the same temperature.

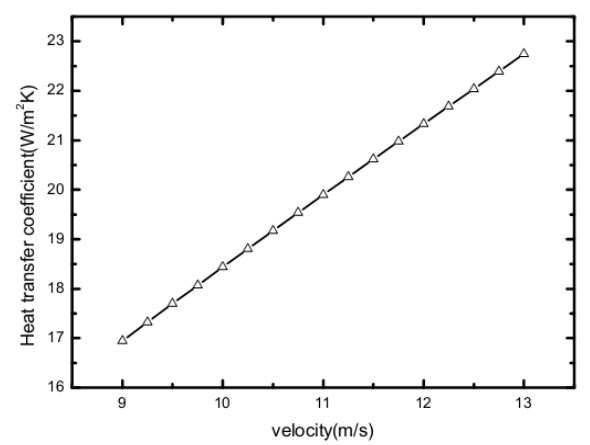

Fig. 5. Variation of outside heat transfer coefficient with the ambient air velocity. 


\subsection{Analysis of thermal performance}

The geometry of wind turbine cabin is similar to cuboids. The wind turbine cabin, which is insulated by polyurethane, foams with its thickness of $15 \mathrm{~mm}$. Cuboids capsule, made of high-strength aluminum, alloys with its thickness of $10 \mathrm{~mm}$. The refrigerant is aqueous ethylene glycol solution of $60 \%$ mass concentration.

This section concerned with the thermal performance of the wind turbine cabin from the numerical point of view. Considering the components layout and boundary conditions, the thermal model is developed by the $\mathrm{VC}++$ program. In addition, the meteorological condition and the material properties are taken into account in the calculation. The results are shown as follows.

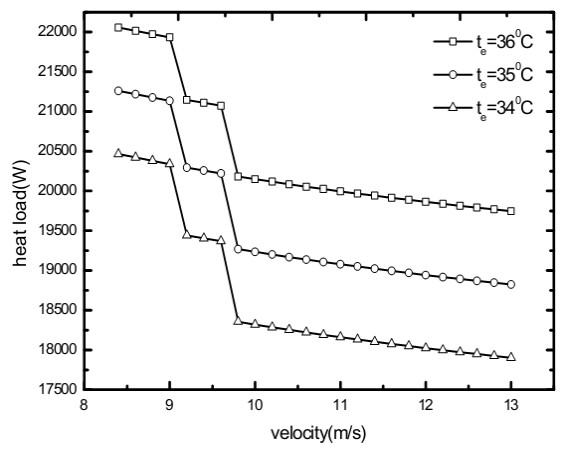

Fig. 6. Variation of the total heat loads with ambient air velocity.

Fig. 6 shows the variation of total heat load of the cabin with the air velocity of ambient environment. It is obtained from the figure that the total heat load of the cabin $Q$ decreases nonlinearly with increasing of the air velocity of ambient environment. And it increases with the ambient temperature if the air velocity of ambient environment $v$ is given. And it is observed that the total heat load decreases with increasing of the air velocity of ambient environment. The results show that the influence of thermal coefficient between the cabin and ambient environment grows larger due to the larger air velocity of ambient environment, which will enhance the heat transfer between the cabin and ambient environment.

Fig. 7 gives the total heat load variation of the cabin with the air velocity of internal cabin air. When the air temperature of ambient environment is $35^{\circ} \mathrm{C}$, the air velocity of ambient environment is $10 \mathrm{~m} / \mathrm{s}$, and the solar radiation intensity is $1353 \mathrm{~W} / \mathrm{m}^{2}$. When the temperature of inside cabin air is $52^{\circ} \mathrm{C}$, the total heat load increases from $10039.8 \mathrm{~W}$ to $16402.5 \mathrm{~W}$ while the inside cabin velocity increases from $1 \mathrm{~m} / \mathrm{s}$ to $2.9 \mathrm{~m} / \mathrm{s}$. And it can be seen from Fig. 7 that the influence of air velocity of inside cabin on the total heat load is large owing to the convection heat transfer coefficient dependent on the air velocity of inside air. It can be also obtained from the Fig. 7 that the total heat load $Q$ decreases nonlinearly with the control temperature of the inside cabin $t_{c}$. The reason is that the 
higher control temperature indicates the smaller temperature difference between the inside cabin air and the surface of the key components, the smaller temperature difference means the weaker heat transfer driving force, which results in the decreasing of the total heat load.

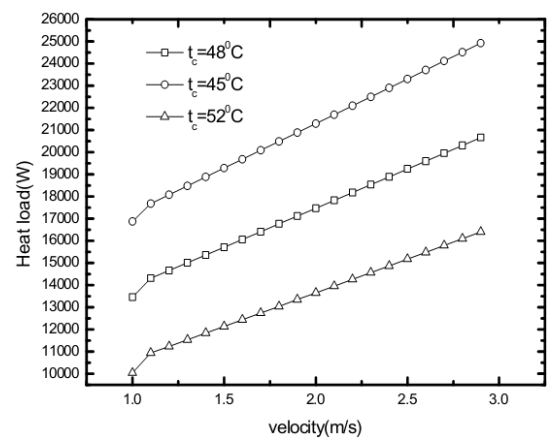

Fig. 7. Variation of the total heat loads with inside cabin air velocity.

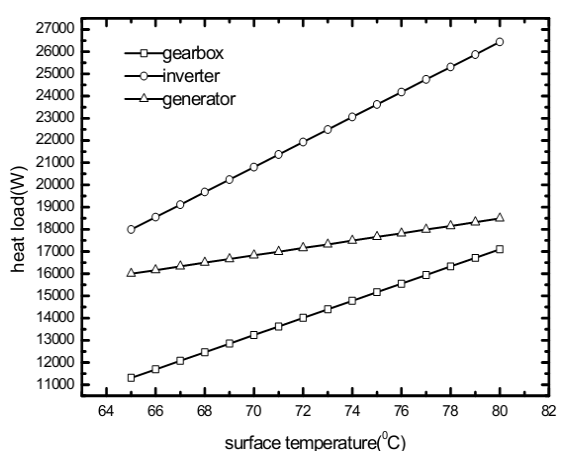

Fig. 8. Variation of the total heat loads with the temperature of key components' surface.

Fig. 8 shows the heat load variation with the temperature of core components' surface. We assume that the ambient environment parameters are constant. It is observed that the heat load linearly increases with the temperature of core components' surface. The heat transfer driving force is larger when the temperature of the core components' surface is larger, which lead to the increase of the inner heat load produced by the core components. It is obvious from Fig. 8 that the variation rate of heat load caused by the inverter is the largest of the three kinds of components in the same scale of temperature variation, which is caused by the largest surface area of the inverter. 


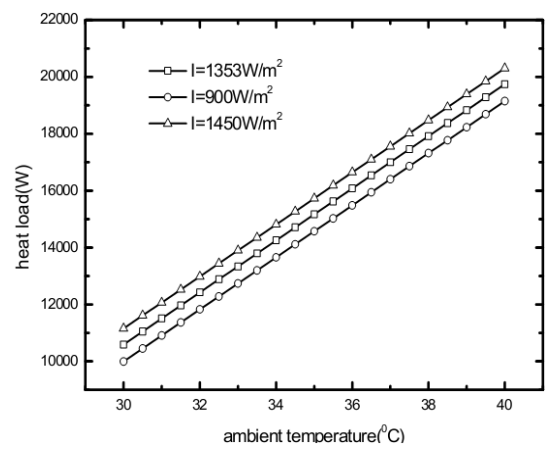

Fig. 9. Variation of the total heat loads with the ambient temperature.

Fig. 9 shows the variation of heat load with ambient environment in different solar radiation absorption rate. From Fig. 9, it is known that at a certain ambient temperature, the heat load increases with the absorption rate of solar radiation. Since the cabin surface temperature increases with the absorption rate of solar radiation, the temperature difference increases with the cabin surface temperature, which results in more heat flux transported from the ambient environment.

Fig. 10 presents the variation of the heat load with time in one day. It is observed from figure that the heat load of the cabin varies obviously with time. Heat load decreases firstly, then increases, and decreases again; the variation trend is the same as the ambient temperature variation with the time. The maximum heat load appears at 14 o'clock while the minimum heat load occurs at about 4 o'clock. The result is determined by the different solar radiation of each hour and the property of the insulation layer.

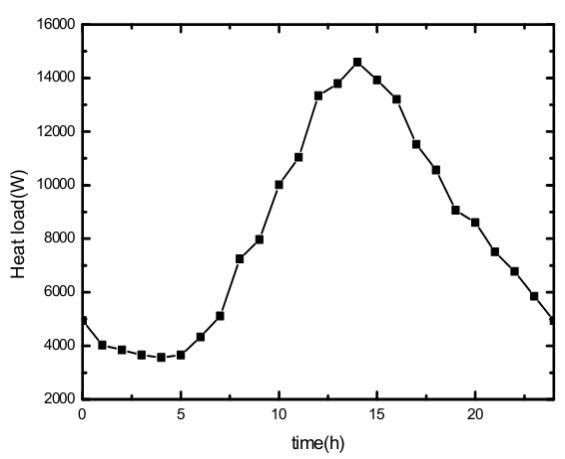

Fig. 10. Variation of the total heat loads with time.

\section{Conclusions}

In this paper, the thermal performance of a $3 \mathrm{MW}$ wind turbine cabin is numerically conducted. The influences of geometrical configuration and physical parameters, the 
meteorological conditions and operation state on thermal behaviors are investigated. The obtained results show that all these factors have a great effect on the heat load of the cabin. As a consequence, to design the cooling system of the wind turbine power generation, not only the heat production of each components should be considered, but also the surface temperature of the core components, the meteorological conditions and the operate state are important parameters. The present work is helpful for the optimization of cooling system in wind turbine.

\section{References}

1. D. Paul, R. Ener. 31, 1355 (2006).

2. J. Shanker, M. P. Sharma and S. S. Kushwah, J. Phys. Chem. Solids, 60, 603 (1999).

3. J. Wang and P. Shao, J. Eng. Design, 12, 315 (2003).

4. K. Taeyeon, K. Shinsuke, M. Shuzo and R. Ji-woong, Bui. Env. 40, 301 (2005).

5. H. Shi, B. Song and L. Zhou, Acta Aero. Et Astro. Sinica, 5, 1 (2009).

6. Y. Wielhorski, P. Mousseau, Y. Jarny, D. Delaunay and N. Lefevre, Int. J. Therm. Sci. 50, 769 (2011).

7. P. Sorensen, A. D. Hansen, P. A. C. Rosas, J. Wind Eng. Ind. Aero. 90, 1381 (2002).

8. W. Yuan and Y. Jiang, World SCI-TECH R\&D, 29, 80 (2007).

9. T. Ni, D. Elec. Rev. 20, 31 (2006).

10. S. Ni, S. Ener. 2, 5 (2000).

11. W. Li, P. Cheng and M. Zhang, Elec. Mach. Cont. 12, 52 (2010).

12. X. Luan, W. Luan and A. D. Sahin, Prog. Ener. Comb. Sci. 30, 501 (2004).

13. P. Li, H. Cheng, App. Therm. Eng. 26, 2020 (2006).

14. N. A. Roberts and D. G. Walker, Int. J. Therm. Sci. 50, 648 (2011). 